\title{
Minimal renal toxicity after Rituximab DHAP with a modified cisplatin application scheme in patients with relapsed or refractory diffuse large B-cell lymphoma
}

K. Lisenko ${ }^{1 *}$, F. McClanahan², T. Schöning ${ }^{3}$, M. A. Schwarzbich ${ }^{1}$, M. Cremer', T. Dittrich ${ }^{1}$, A. D. Ho ${ }^{1}$ and M. Witzens-Harig ${ }^{1}$

\begin{abstract}
Background: Rituximab (R) in combination with DHAP is a widely accepted salvage regimen for patients with relapsed or refractory diffuse large B-cell lymphoma (DLBCL). A common adverse effect of this protocol is renal toxicity which may result in treatment discontinuation. Assuming that a lower single dose of cisplatin over several days would reduce renal toxicity, our institution has chosen to administer cisplatin in a dosage of $25 \mathrm{mg} / \mathrm{m}^{2}$ per day as a 3-h infusion over 4 consecutive days.
\end{abstract}

Methods: In this study, we analyzed the renal function of 122 patients with relapsed or refractory DLBCL treated with R-DHAP at our institution. Overall, 256 R-DHAP cycles were administered. 31 (25\%), 61 (50 \%), 14 (12\%) and 16 (13\%) patients received one, two, three or four R-DHAP courses, respectively.

Results: A glomerular filtration rate (GFR) decrease was observed after each R-DHAP cycle. However, in none of the subgroups the median GFR was lower than $60 \mathrm{ml} / \mathrm{min} / 1.73 \mathrm{~m}^{2}$. In most patients, only renal impairment stage I and II was observed. Renal impairment stage III was seen in $10 \%$ and stage IV only in $1 \%$ of patients.

Conclusion: We conclude that a modified R-DHAP regimen with administration of cisplatin $25 \mathrm{mg} / \mathrm{m}^{2}$ over 4 consecutive cycles leads only to minimal renal toxicity.

Keywords: R-DHAP, DLBCL, Renal chemo toxicity

\section{Background}

The addition of the anti-CD20 monoclonal antibody rituximab (R) have significantly improved the outcome and survival of patients with diffuse large B-cell lymphoma (DLBCL), and complete responses (CRs) after first line therapy are observed in 75-80 \% [1-3]. However, approximately one-third of patients develop relapsed or refractory disease [4]. For eligible patients, inductionchemoimmunotherapy, and in case of chemosensitive disease, high-dose (HD) chemotherapy and autologous stem cell transplantation (ASCT) are the current standard of care [5]. Such salvage therapies typically consist of

\footnotetext{
* Correspondence: katharina.lisenko@med.uni-heidelberg.de 'Department of Hematology, Oncology and Rheumatology, University Hospital Heidelberg, Im Neuenheimer Feld 410, Heidelberg 69120, Germany Full list of author information is available at the end of the article
}

cytotoxic agents that have not been used in first line therapy. One established regime in relapsed or refractory DLBCL is R-DHAP. R-DHAP combines rituximab $\left(375 \mathrm{mg} / \mathrm{m}^{2}\right)$, given intravenously (i.v.) one day before chemotherapy, cisplatin $\left(100 \mathrm{mg} / \mathrm{m}^{2}\right)$, typically administered i.v. by continuous infusion over $24 \mathrm{~h}$, followed on day 2 by cytarabine $\left(2 \mathrm{~g} / \mathrm{m}^{2}\right)$ in a 3-h long infusion (repeated after $12 \mathrm{~h}$ ), and oral dexamethasone $(40 \mathrm{mg} / \mathrm{d}$ for 4 consecutive days) repeated after 21 days for 2-4 cycles. However, this regimen is associated with severe adverse effects that often require discontinuation of treatment, such as myelosuppression, infections and renal toxicity. For instance, in a phase II trial in relapsed non-Hodgkin lymphoma evaluating the toxicity and efficacy of adding 4 doses of rituximab to a conventional DHAP regimen grade III or IV nephrotoxicity was observed in $7 \%$ of 
patients after two R-DHAP courses [6]. Moreover, in a randomized phase III trial in relapsed or refractory DLBCL comparing two salvage regimes, stage IV renal toxicity occurred in $6 \%$ of patients treated with three cycles of conventional R-DHAP [7]. Because of the efficacy of the R-DHAP regimen, attempt to reduce nephrotoxicity e. g. by substitution of cisplatin with oxaliplatin has been successfully made [8]. Another attractive approach to limit renal toxicity is to spread the cisplatin application over several days. For example, cisplatin can be administered for 4 consecutive days as a $3 \mathrm{~h}$ infusion, consisting of $25 \mathrm{mg} / \mathrm{m}^{2}$ each. Moreover, the administration of cisplatin for several hours on four consecutive days, instead of a $24 \mathrm{~h}$ infusion, allows treating patients with this regimen on an outpatient basis. However, a systematic evaluation of the toxicity and efficacy of this modification is warranted. In the current study we therefore retrospectively analyzed 122 patients with relapsed or refractory DLBCL treated with the modified R-DHAP regimen at our institution. Our aims were to evaluate the efficacy and renal toxicity of this salvage therapy regimen.

\section{Methods}

\section{Patient selection}

All patients with relapsed or refractory DLBCL that were treated with R-DHAP at our institution from July 2002 to July 2013 received the modified R-DHAP regimen, under a hypothetical, but so far not verified, assumption that a lower single dose of cisplatin over several days would reduce renal toxicity. Overall, 122 patients were included into this analysis. All patients had histologically confirmed DLBCL. Clinical characteristics (age, gender, stage at diagnosis), previous therapy, time to relapse and serum creatinine before each individual and after the last R-DHAP course were collected and retrospectively analyzed. This analysis was approved by the ethics committee University Hospital Heidelberg without an informed consent of the patients with regard to its retrospectivity. Research was carried out in compliance with the Helsinki Declaration.

\section{Modified R-DHAP regimen}

Rituximab $\left(375 \mathrm{mg} / \mathrm{m}^{2}\right)$ was administered i.v. on day 1 using standard supportive therapy. Dexamethasone $(40 \mathrm{mg}$ ) was given orally for 4 consecutive days. Cytarabine $\left(2 \mathrm{~g} / \mathrm{m}^{2}\right)$ was administered on day 2 as a 3-h infusion and was repeated after $12 \mathrm{~h}$. Cisplatin $\left(25 \mathrm{mg} / \mathrm{m}^{2}\right)$ was applied on days 1 to 4 as a 3-h infusion. Dose modifications were implemented according to renal function as follows: At a glomerular filtration rate (GFR) below $60 \mathrm{ml} / \mathrm{min} / 1.73 \mathrm{~m}^{2}$, cytarabine was reduced to $75 \%$ of the original dose, and to $50 \%$ at a GFR below $50 \mathrm{ml} /$ $\min / 1.73 \mathrm{~m}^{2}$. Cisplatin was reduced to $60 \%$ and $50 \%$, respectively. A GFR below $30 \mathrm{ml} / \mathrm{min} / 1.73 \mathrm{~m}^{2}$ was considered a contraindication for cytarabine und cisplatin. Cycles were repeated every three weeks. For prevention of chemotherapy-induced nausea and vomiting, intensified oral supportive medication (dexamethasone $8 \mathrm{mg}$ and granisetron hydrochloride $2 \mathrm{mg}$ days 1 to 4 , aprepitant $125 \mathrm{mg}$ day 1 , aprepitant $80 \mathrm{mg}$ day 1 to 6) was used [9].

\section{Assessment of clinical responses}

Response to R-DHAP was evaluated by clinical examination and computed tomography scan of the involved lymph node regions according to standardized response criteria for non-Hodgkin lymphomas [10].

\section{Assessment of renal toxicity}

Renal toxicity was assessed by measuring serum creatinine levels up to three days before each R-DHAP cycle and two to three weeks after termination of each RDHAP cycle. GFR was calculated according to the Chronic Kidney Disease Epidemiology Collaboration (CKD-EPI) equation [11]. GFR above $90 \mathrm{ml} / \mathrm{min} / 1.73 \mathrm{~m}^{2}$ was considered normal renal function. GFRs of 89-60, $59-30,29-15$ and $<15 \mathrm{ml} / \mathrm{min} / 1.73 \mathrm{~m}^{2}$ were graded as stage II, III, IV or V renal function impairment.

\section{Statistical analysis}

Patients were grouped according to the overall number of received R-DHAP courses (minimum one, maximum four). Descriptive statistics and comparison between the groups was performed by Prism 5.03. Data are given as absolute numbers and percentage, and if not otherwise stated as mean and standard deviation (SD). To increase visual evidence and to allow better comparison, GFR levels are described as mean and SD in the text and illustrated as box plots (minimum, first quartile, median, third quartile and maximum, Fig. 1 and 3). For comparison of categorical variables (gender, stage at first diagnosis, previous therapy, time to relapse after last treatment and response to R-DHAP) Freeman-Halton extension of Fisher's Exact test was used. To identify differences among groups' means (age at first diagnosis, age at first R-DHAP and duration between last treatment and first R-DHAP) an analysis of variance was performed. Mean differences in individual pairwise comparisons of preand post-treatment GFR values were made using paired two tailed student's t test. A $P<0.05$ was considered statistically significant.

\section{Results}

\section{Patient characteristics}

A total of 122 patients with relapsed or refractory DLBCL were treated with R-DHAP as second-line therapy at our institution. The mean age at first R-DHAP 


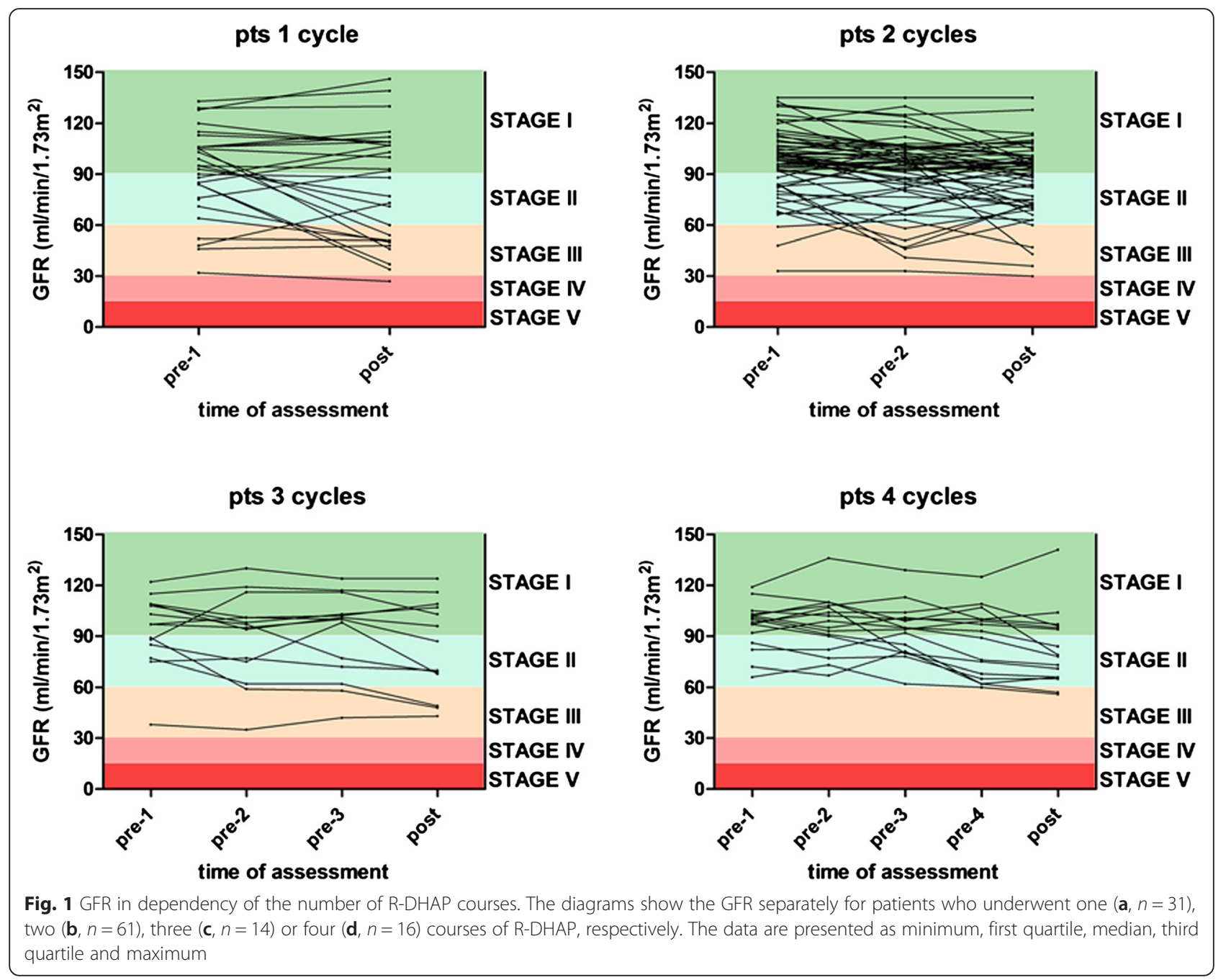

was 57 (SD 12) years, and the male:female ratio was 2:1. 113 (93\%) patients had previously received CHOP-like treatment. In 96 (79 \%) patients, first line therapy included rituximab. Most patients had progressive disease or relapsed within 12 months after first treatment. Detailed patient characteristics and treatment details are summarized in Table 1.

\section{Exposure and response to R-DHAP treatment}

Overall, 256 R-DHAP cycles were administered. 31 (25\%), 61 (50\%), 14 (12\%) and 16 (13\%) patients received one, two, three or four R-DHAP courses, respectively. Dose adjustments were applied in only 5 (4\%) patients who in total received 11 R-DHAP courses. In one patient cytarabine was reduced to $50 \%$ due to advanced age ( $>70$ years) and poor general condition. One patient received carboplatin instead of cisplatin due to stage III renal impairment. In two patients, both cytarabine and cisplatin dose adjustments were necessary due to a GFR $<50 \mathrm{ml} / \mathrm{min} / 1.73 \mathrm{~m}^{2}$. In one patient, reasons for and extent of R-DHAP dose adjustments were not documented. In 5 patients the response to R-DHAP treatment was not available. 19 (16\%), 6 (5\%), 39 (32\%) and 18 (15\%) patients achieved CR, CRu (complete response unconfirmed), partial remission (PR) and stable disease $(\mathrm{StD})$, respectively. $35(29 \%)$ patients had progressive disease (PD), which predominantly occurred after the first two cycles of R-DHAP (Table 1).

\section{Renal toxicity}

In patients who received one cycle of R-DHAP the initial mean GFR was 91 (SD 25) $\mathrm{ml} / \mathrm{min} / 1.73 \mathrm{~m}^{2} .17$ (55\%) patients had normal renal function. Ten (32\%) and four (13\%) patients showed an initial stage II and III renal impairment. After one course of R-DHAP, the GFR remained stable (mean 84 , SD $34 \mathrm{ml} / \mathrm{min} / 1.73 \mathrm{~m}^{2}, P=$ 0.06 , Fig. 1a). We detected individual deterioration in renal function by one toxicity stage in nine patients and by two toxicity stages in two patients (Fig. 2a). 
Table 1 Patient characteristics and response to R-DHAP

\begin{tabular}{|c|c|c|c|c|c|c|}
\hline & \multirow{2}{*}{$\begin{array}{l}\text { Overall } \\
\text { cohort }\end{array}$} & \multirow{2}{*}{$\begin{array}{l}1 \text { cycle } \\
\text { R-DHAP }\end{array}$} & \multirow{2}{*}{$\begin{array}{l}2 \text { cycles } \\
\text { R-DHAP }\end{array}$} & \multirow{2}{*}{$\begin{array}{l}3 \text { cycles } \\
\text { R-DHAP }\end{array}$} & \multirow{2}{*}{$\begin{array}{l}4 \text { cycles } \\
\text { R-DHAP }\end{array}$} & \multirow[t]{2}{*}{$P$-values } \\
\hline & & & & & & \\
\hline Patients, n (\%) & $122(100)$ & $31(25)$ & $61(50)$ & $14(12)$ & $16(13)$ & / \\
\hline Gender, n (\%) & & & & & & 0.17 \\
\hline Male & $82(67)$ & $20(65)$ & $37(61)$ & $11(79)$ & $14(88)$ & \\
\hline Female & $40(33)$ & $11(35)$ & $24(39)$ & $3(21)$ & $2(12)$ & \\
\hline \multicolumn{7}{|l|}{ First diagnosis and previous Tx } \\
\hline Mean age at first diagnosis (SD) & $53(12)$ & $53(13)$ & $54(11)$ & $51(13)$ & $54(12)$ & 0.73 \\
\hline Stage at first diagnosis, n (\%) & & & & & & 0.01 \\
\hline I & $7(6)$ & $1(3)$ & $2(3)$ & $4(29)$ & $0(0)$ & \\
\hline$\|$ & $26(21)$ & $4(13)$ & $14(23)$ & $3(21)$ & $5(31)$ & \\
\hline III & $39(32)$ & $12(39)$ & $19(31)$ & $3(21)$ & $5(31)$ & \\
\hline IV & $48(39)$ & $13(42)$ & $26(43)$ & $3(21)$ & $6(38)$ & \\
\hline Not known & $2(2)$ & $1(3)$ & $0(0)$ & $1(7)$ & $0(0)$ & \\
\hline CHOP-like therapy, n (\%) & $113(93)$ & $29(94)$ & $58(95)$ & $12(86)$ & $14(88)$ & 0.35 \\
\hline Other, n (\%) & $8(6)$ & $2(6)$ & $2(3)$ & $2(14)$ & $2(12)$ & \\
\hline Not known, n (\%) & $1(1)$ & $0(0)$ & $1(2)$ & $0(0)$ & $0(0)$ & \\
\hline Rituximab-containing, n (\%) & $96(79)$ & $30(97)$ & $47(77)$ & $10(71)$ & $9(56)$ & 0.01 \\
\hline \multicolumn{7}{|l|}{ Relapse } \\
\hline TTR after last treatment, n (\%) & & & & & & 0.01 \\
\hline Refractory or $<12$ months & $79(65)$ & $26(84)$ & $40(66)$ & $6(43)$ & $7(44)$ & \\
\hline$\geq 12$ months & $43(35)$ & $5(16)$ & $21(34)$ & $8(57)$ & $9(56)$ & \\
\hline Mean age at 1. R-DHAP (SD) & $57(12)$ & $56(15)$ & $57(11)$ & $56(13)$ & $57(13)$ & 0.98 \\
\hline Months between last treatment and 1. R-DHAP, mean (SD) & $19(29)$ & $8(15)$ & $17(28)$ & $43(47)$ & $27(23)$ & $<0.01$ \\
\hline Response to R-DHAP, n (\%) & & & & & & $<0.01$ \\
\hline$C R$ & $19(16)$ & $0(0)$ & $11(18)$ & $5(36)$ & $3(19)$ & \\
\hline $\mathrm{CRu}$ & $6(5)$ & $0(0)$ & $5(8)$ & $0(0)$ & $1(6)$ & \\
\hline PR & $39(32)$ & $3(10)$ & $18(30)$ & $6(43)$ & $12(75)$ & \\
\hline $\mathrm{StD}$ & $18(15)$ & $5(16)$ & $11(18)$ & $2(14)$ & $0(0)$ & \\
\hline PD & $35(29)$ & $18(58)$ & $16(26)$ & $1(7)$ & $0(0)$ & \\
\hline Not known & $5(4)$ & $5(16)$ & $0(0)$ & $0(0)$ & $0(0)$ & \\
\hline
\end{tabular}

CHOP cyclophosphamide, hydroxydaunorubicin, vincristine, prednisone, $C R(u)$ complete response (unconfirmed), $P B S C$ peripheral blood stem cell collection, $P D$ progressive disease, $P R$ partial remission, $R-D H A P$ rituximab, dexamethasone, cytarabine, cisplatin, $S t D$ stable disease, $S D$ standard deviation, $T R R$ time to relapse. Statistically significant $P$-values are indicated in bold.

In patients who received two courses of R-DHAP, the initial mean GFR significantly decreased from 97 (SD 20) $\mathrm{ml} / \mathrm{min} / 1.73 \mathrm{~m}^{2}$ to $92(\mathrm{SD} 22) \mathrm{ml} / \mathrm{min} / 1.73 \mathrm{~m}^{2}$ after the first chemotherapy cycle $(P<0.01)$ and to 88 (SD 20) $\mathrm{ml} / \mathrm{min} / 1.73 \mathrm{~m}^{2}$ after the second course of $\mathrm{R}$ DHAP $(P<0.001$, Fig. 1b). Before treatment, $42(69 \%)$ patients had normal renal function. Eight of these patients had worsening kidney function by one stage and one by two stages. In all but one of $15(25 \%)$ patients who showed stage II renal impairment before R-DHAP kidney function remained stable or even improved after two courses of therapy. In three (5\%) patients who had stage III renal function impairment before therapy, no decrease in renal function was observed (Fig. 2b). In one patient initial renal function was not available.

A significant GFR decrease in patients who have been treated with three R-DHAP courses from initially 94 (SD 21) to 84 (SD 27) $\mathrm{ml} / \mathrm{min} / 1.73 \mathrm{~m}^{2}$ after the third course of therapy $(P=0.04)$ was observed. While the GFR was almost stable over the first two cycles of chemotherapy the main decline occurred after the third R-DHAP cycle, where the mean GFR fell by $7 \mathrm{ml} / \mathrm{min} / 1.73 \mathrm{~m}^{2}$ (Fig. 1c). We noticed a worsening in renal function from stage I to stage II in three patients and from stage II to stage III in two patient of this subgroup (Fig. 2c). 

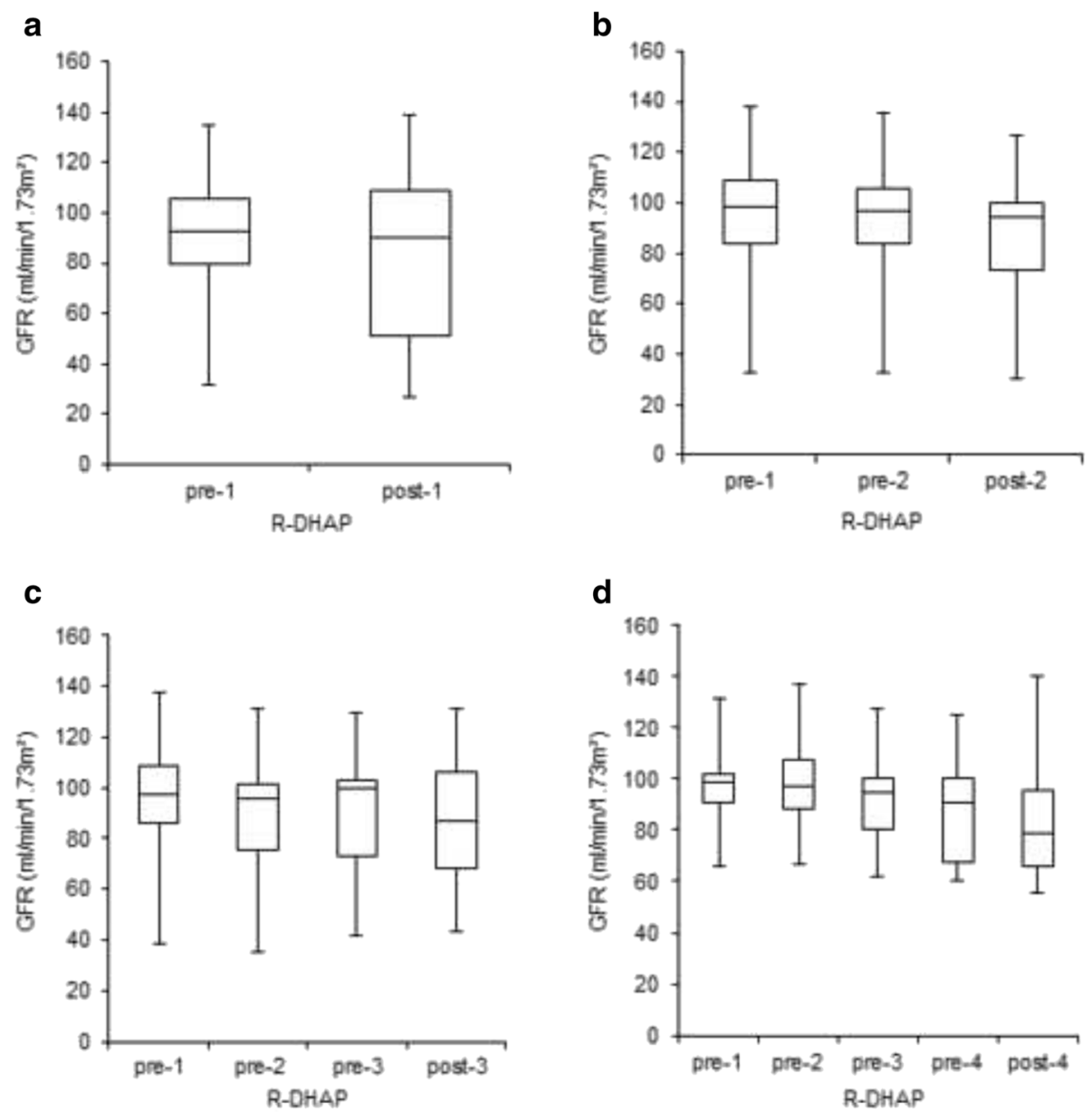

Fig. 2 Renal function during R-DHAP treatment by stage. GFR and corresponding renal function by stage during the course of therapy is shown separately for every single patient who underwent one $(\mathbf{a}, n=31)$, two $(\mathbf{b}, n=61)$, three $(\mathbf{c}, n=14)$ or four $(\mathbf{d}, n=16)$ cycles of R-DHAP

Similarly, in patients who received four R-DHAP cycles, the initial mean GFR of 96 (SD 14) $\mathrm{ml} / \mathrm{min} / 1.73 \mathrm{~m}^{2}$ remained stable over the first two cycles. After the third and fourth course of R-DHAP, the mean GFR significantly fell to 87 (SD 20, $P=0.01$ ) and 83 (SD 22, $P<$ $0.01) \mathrm{ml} / \mathrm{min} / 1.73 \mathrm{~m}^{2}$ (Fig. 1d). In this subgroup we found a decrease in renal function from stage I to stage II in six patients and from stage II to stage III in two patients (Fig. 2d).

Figure 3 summarizes the GFR separated by subgroup in dependency of the course of R-DHAP therapy.

\section{Discussion}

We here performed an analysis of 122 patients with relapsed or refractory DLBCL treated with a modified RDHAP regimen. Contrasting the practice of other centers, the total dose of cisplatin was maintained but spread over 4 consecutive days to minimize renal toxicity. Our aim was to assess renal toxicity associated with this regimen using a "real-life" population not selected for clinical trials. This series was homogeneous according to the diagnosis relapsed or refractory DLBCL, gender distribution, previous CHOP-like therapy and age at first R-DHAP but heterogeneous according to stage at first diagnosis, time to elapse after last treatment and duration between last treatment and first R-DHAP among the four R-DHAP groups.

We show that the modified R-DHAP regimen as administered in our institution is efficient and safe in this patient group. We observed chemosensitivity in $68 \%$ of patients, and PD occurred in only one third of the patients. This is in accordance with response rates reported for conventional R-DHAP regimens applied in prospective trials, where response to therapy was observed in $76 \%$ of the patients (7).

Importantly, modified R-DHAP did not lead to severe renal impairment in our cohort. When analyzing GFR according to the number of cycles received, renal impairment stage III was observed in less than $10 \%$ and stage IV only in less than $1 \%$ of patients, and none of 


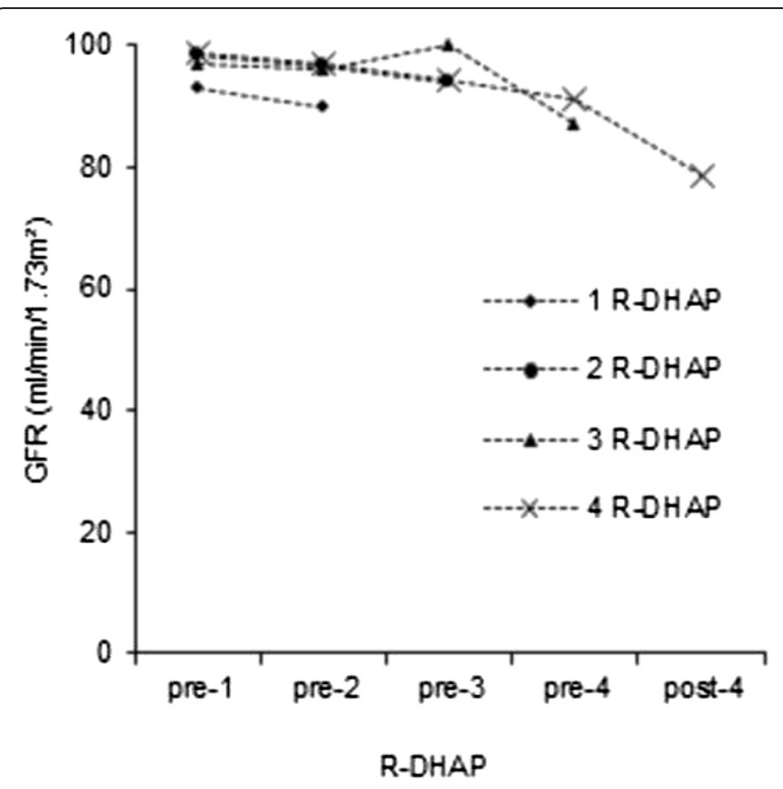

Fig. 3 Comparison of GFR between the subgroups. The diagram summarizes the development of median GFR in the course of therapy subdivided by the number of R-DHAP cycles received

the subgroups had an initial average GFR of less than $60 \mathrm{ml} / \mathrm{min} / 1.73 \mathrm{~m}^{2}$. Within subgroups we found an additive renal toxicity over the course of R-DHAP treatment, but a statistically significant GFR decrease was observed only after the third and fourth R-DHAP course. A direct prospective comparison between modified and conventional R-DHAP regimens has not been conducted yet and unfortunately, comparisons of retrospective results published by different centers on non-modified R-DHAP are hampered by the heterogeneity of patient cohorts and treatment approaches [7, 8, 12, 13].

In particular, renal toxicity was not assessed in detail and in relation to the number of treatment cycles received the majority of published studies using conventional R-DHAP. In a randomized phase III trial in relapsed or refractory DLBCL comparing the salvage regimes R-DHAP versus R-ICE (ifosfamide, carboplatin, etoposide), stage IV renal toxicity was observed in 11 of 194 patients $(6 \%)$ treated with conventional R-DHAP over the course of three cycles (7). In contrast, we observed no stage IV renal toxicity in patients who received three or even four courses of modified R-DHAP.

To the best of our knowledge this is the first detailed evaluation of renal function during R-DHAP treatment in a large series of relapsed or refractory DLBLC patients. Our data show that the administration of $25 \mathrm{mg} / \mathrm{m}^{2}$ cisplatin for 4 consecutive days in a $3 \mathrm{~h}$ infusion as a component of the R-DHAP regimen is efficient in relapsed or refractory DLBCL and leads to a tolerable additive renal toxicity over up to four R-DHAP courses. Furthermore, this study demonstrates that a detailed analysis of renal function impairment after each chemotherapy cycle is required to assure comparability between different chemotherapy protocols.

\section{Conclusion}

R-DHAP (rituximab, cisplatin, cytarabine, dexamethasone) is an accepted salvage regimen for relapsed or refractory diffuse large B-cell lymphoma patients. Assuming that a lower single dose of cisplatin over several days would reduce renal toxicity, the total dose of cisplatin $\left(100 \mathrm{mg} / \mathrm{m}^{2}\right)$ was spread over 4 consecutive days. Renal impairment stage III was seen in $10 \%$ and stage IV only in $1 \%$ of patients indicating minimal renal toxicity of the modified R-DHAP regimen.

\section{Abbreviations}

ASCT: autologous stem cell transplantation; CKD-EPI: Chronic Kidney Disease Epidemiology Collaboration; CHOP: cyclophosphamide, hydroxydaunorubicin, vincristine, prednisone; $C R(u)$ : complete response (unconfirmed); DHAP: dexamethasone, cytarabine, cisplatin; DLBCL: diffuse large B-cell lymphoma; GFR: glomerular filtration rate; HD: high-dose; i.v.: intravenous; PBSC: peripheral blood stem cell collection; PD: progressive disease; PR: partial remission; R: Rituximab; SD: standard deviation; StD: stable disease; TTR: time to relapse.

\section{Competing interest}

Mathias Witzens-Harig: consultancy for Celgene and honorarium from Roche. Anthony D. Ho: consultancy, honoraria and membership on Advisory Boards of Genzyme/Sanofi-Aventis. All other authors have nothing to disclose.

\section{Authors' contributions}

$\mathrm{KL}, \mathrm{FM}$, and $\mathrm{MW}-\mathrm{H}$ have made substantial contributions to conception and design, or acquisition of data, or analysis and interpretation of data. TS, MAS, $M C, T D$, and $A D H$ have been involved in drafting the manuscript or revising it critically for important intellectual content. TS, MAS, MC, TD, and ADH have given final approval of the version to be published. All authors have read and approved the final version of the manuscript.

\section{Acknowledgements}

All contributors meet the criteria for authorship.

\section{Source of funding}

None.

\section{Author details}

'Department of Hematology, Oncology and Rheumatology, University Hospital Heidelberg, Im Neuenheimer Feld 410, Heidelberg 69120, Germany. ${ }^{2}$ Centre for Haemato-Oncology/Barts Cancer Institute, Queen Mary University of London, London, UK. ${ }^{3}$ Pharmacy Department, University Hospital Heidelberg, Heidelberg, Germany.

Received: 2 November 2015 Accepted: 22 March 2016

Published online: 11 April 2016

References

1. Pfreundschuh M, Schubert J, Ziepert M, Schmits R, Mohren M, Lengfelder E, et al. Six versus eight cycles of bi-weekly CHOP-14 with or without rituximab in elderly patients with aggressive CD20+ B-cell lymphomas: a randomised controlled trial (RICOVER-60). Lancet Oncol. 2008;9(2):105-16.

2. Habermann TM, Weller EA, Morrison VA, Gascoyne RD, Cassileth PA, Cohn $J B$, et al. Rituximab-CHOP versus $\mathrm{CHOP}$ alone or with maintenance rituximab in older patients with diffuse large B-cell lymphoma. J Clin Oncol. 2006;24(19):3121-7.

3. Coiffier B, Lepage E, Briere J, Herbrecht R, Tilly H, Bouabdallah R, et al. CHOP chemotherapy plus rituximab compared with CHOP alone in elderly patients with diffuse large-B-cell lymphoma. N Engl J Med. 2002;346(4):235-42. 
4. Friedberg JW. Relapsed/refractory diffuse large B-cell lymphoma. Hematology/the Education Program of the American Society of Hematology. Am Soc Hematol Educ Program. 2011;2011:498-505.

5. Philip T, Guglielmi C, Hagenbeek A, Somers R, Van der Lelie H, Bron D, et al. Autologous bone marrow transplantation as compared with salvage chemotherapy in relapses of chemotherapy-sensitive non-Hodgkin's lymphoma. N Engl J Med. 1995;333(23):1540-5.

6. Witzig TE, Geyer SM, Kurtin PJ, Colgan JP, Inwards DJ, Micallef IN, et al. Salvage chemotherapy with rituximab DHAP for relapsed non-Hodgkin lymphoma: a phase II trial in the North Central Cancer Treatment Group. Leuk Lymphoma. 2008:49(6):1074-80.

7. Gisselbrecht C, Glass B, Mounier N, Singh Gill D, Linch DC, Trneny M, et al. Salvage regimens with autologous transplantation for relapsed large B-cell lymphoma in the rituximab era. J Clin Oncol. 2010;28(27):4184-90.

8. Lignon J, Sibon D, Madelaine I, Brice P. Franchi P, Briere J, et al. Rituximab, dexamethasone, cytarabine, and oxaliplatin (R-DHAX) is an effective and safe salvage regimen in relapsed/refractory B-cell non-Hodgkin lymphoma. Clin Lymphoma Myeloma Leuk. 2010;10(4):262-9.

9. Schmitt T, Goldschmidt H, Neben K, Freiberger A, Husing J, Gronkowski M, et al. Aprepitant, granisetron, and dexamethasone for prevention of chemotherapy-induced nausea and vomiting after high-dose melphalan in autologous transplantation for multiple myeloma: results of a randomized, placebo-controlled phase III trial. J Clin Oncol. 2014;32(30):3413-20.

10. Cheson BD, Horning SJ, Coiffier B, Shipp MA, Fisher Rl, Connors JM, et al. Report of an international workshop to standardize response criteria for non-Hodgkin's lymphomas. NCI Sponsored International Working Group. J Clin Oncol. 1999;17(4):1244.

11. Levey AS, Stevens LA, Schmid CH, Zhang YL, Castro 3rd AF, Feldman HI, et al. A new equation to estimate glomerular filtration rate. Ann Intern Med. 2009;150(9):604-12.

12. Kanat O, Ozet A, Ataergin S, Arpaci F, Kuzhan O, Komurcu S, et al. Modified outpatient dexamethazone, cytarabine and cisplatin regimen may lead to high response rates and low toxicity in lymphoma. Med Princ Pract. 2010; 19(5):344-7.

13. Velasquez WS, Cabanillas F, Salvador P, McLaughlin P, Fridrik M, Tucker S, et al. Effective salvage therapy for lymphoma with cisplatin in combination with high-dose Ara-C and dexamethasone (DHAP). Blood. 1988;71(1):117-22.

\section{Submit your next manuscript to BioMed Central and we will help you at every step:}

- We accept pre-submission inquiries

- Our selector tool helps you to find the most relevant journal

- We provide round the clock customer support

- Convenient online submission

- Thorough peer review

- Inclusion in PubMed and all major indexing services

- Maximum visibility for your research

Submit your manuscript at www.biomedcentral.com/submit

C Biomed Central 